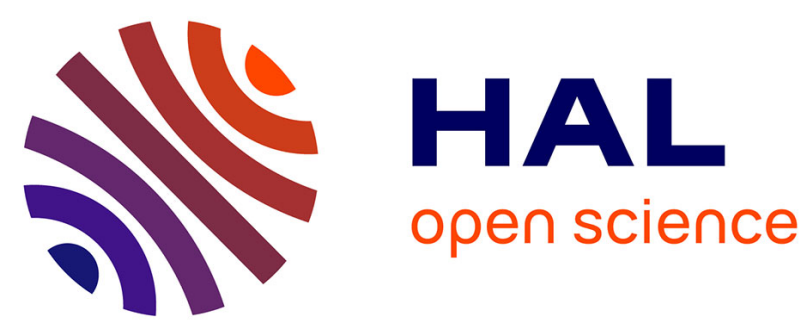

\title{
Interactive 3D brain vessel segmentation from an example
}

\author{
Alice Dufour, Nicolas Passat, Benoît Naegel, Joseph Baruthio
}

\section{To cite this version:}

Alice Dufour, Nicolas Passat, Benoît Naegel, Joseph Baruthio. Interactive 3D brain vessel segmentation from an example. International Symposium on Biomedical Imaging (ISBI), 2011, Chicago, United States. pp.1121-1124, 10.1109/ISBI.2011.5872598 . hal-01695036

\section{HAL Id: hal-01695036 \\ https://hal.univ-reims.fr/hal-01695036}

Submitted on 3 Mar 2018

HAL is a multi-disciplinary open access archive for the deposit and dissemination of scientific research documents, whether they are published or not. The documents may come from teaching and research institutions in France or abroad, or from public or private research centers.
L'archive ouverte pluridisciplinaire HAL, est destinée au dépôt et à la diffusion de documents scientifiques de niveau recherche, publiés ou non, émanant des établissements d'enseignement et de recherche français ou étrangers, des laboratoires publics ou privés. 


\title{
INTERACTIVE 3D BRAIN VESSEL SEGMENTATION FROM AN EXAMPLE
}

\author{
Alice Dufour ${ }^{1,2}$, Nicolas Passat ${ }^{1}$, Benoît Naegel ${ }^{3}$ and Joseph Baruthio ${ }^{2}$ \\ ${ }^{1}$ Université de Strasbourg, LSIIT, UMR CNRS 7005, France \\ ${ }^{2}$ Université de Strasbourg, LINC, FRE CNRS 3289, France \\ ${ }^{3}$ Université Nancy I, LORIA, UMR CNRS 7503, France
}

\begin{abstract}
Segmentation of cerebral vascular networks from 3D angiographic data remains a challenge. Automation generally induces a high computational cost and possible errors, while interactive methods are hard to use due to the dimension and complexity of images. This article presents a compromise between both approaches, by using the concept of examplebased segmentation. Segmentation examples of vascular structures are involved in a scheme relying on connected filtering, in order to obtain an interactive -but strongly assistedsegmentation method. This strategy, which uses componenttrees in a non-standard fashion, leads to promising results, when applied on cerebral MR angiographic data.
\end{abstract}

Index Terms - Vessel segmentation, example-based segmentation, mathematical morphology, MRA.

\section{INTRODUCTION}

Cerebrovascular segmentation is used for several purposes, including vascular pathology diagnosis and quantification, or neurosurgery planning. The specific properties of vessels (small structures w.r.t. the image resolution) and of angiographic data (sparseness, low SNR when non-injected, morphological artifacts, etc.) justify that their segmentation remains a challenge.

Several techniques have been considered for designing vessel segmentation methods, especially devoted to 3D data, including in particular Magnetic Resonance Angiography (MRA). One can cite, non exhaustively, deformable models, vessel tracking, statistical analysis, and mathematical morphology. (A state of the art is beyond the scope of this article, a recent and complete survey may be found in [1].) Usually, these methods are globally automatic (except, sometimes, for initialization and/or termination, or for the determination of parameters). Consequently, they can hardly take advantage of the expertise of the user (clinician, radiologist, etc.), thus leading to possible segmentation errors (in addition to a frequently high computational cost). Interactive methods, which could a contrario rely on the user skills, are generally not

The research leading to these results has been partially funded by a $\mathrm{PhD}$ grant (A. Dufour) of the Région Alsace and CNRS. considered due to the size and complexity of the 3D images to be processed.

However, a compromise between automatic and interactive segmentation could be proposed, thanks to the notion of example-based segmentation, which has been considered in several application fields $[2,3]$ before being applied to medical imaging [4]. The use of segmentation examples can lead to an automatic presegmentation of the regions where are located the vessels, and in particular to determine a subnetwork of interest into the vascular network, e.g., for vein/artery separation in images where both structures appear.

Methodologies based on mathematical morphology have been recently considered for medical image segmentation, especially in the case of angiographic images [5]. Among the involved techniques, the ones based on connected filtering, and more especially on component-trees [6] have led to promising results [7]. Component-trees are adapted to process grey-scale images, where the structures of interest have properties relating to the topology (connectivity) and intensity (local/global extrema). They are essentially used for filtering purpose, by considering the selection of a part of the component-tree corresponding to nodes having properties in adequacy with criteria modeling the searched objects.

In this work, we propose to use the component-tree in a different fashion by searching a set of nodes generating a resulting image being as close as possible to a segmentation example, w.r.t. a false positive/negative cost function. This non-standard strategy enables in particular to fuse component-trees and example-based segmentation.

This article is organized as follows. Sec. 2 presents theoretical and methodological concepts of the proposed segmentation technique. Experimental results are presented in Sec. 3. A discussion and perspectives will be found in Sec. 4 .

\section{METHOD}

\subsection{Definitions and Notations}

Let $I: E \rightarrow V\left(E \in \mathbb{Z}^{n}\right.$, and $\left.V=[0, m]\right)$ be a greylevel image (we also note $I \in V^{E}$ ). For $X \subseteq E$, let $\mathcal{C}[X]$ be the set of the connected components of $X$, induced by a chosen adjacency relation. For any $v \in V$, we define the 


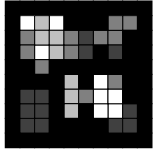

(a)

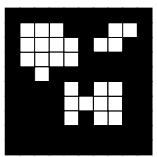

(d)

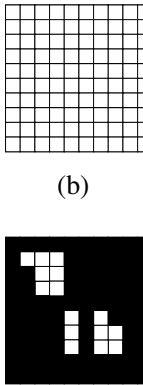

(e)

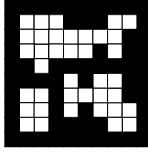

(c)

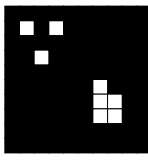

(f)

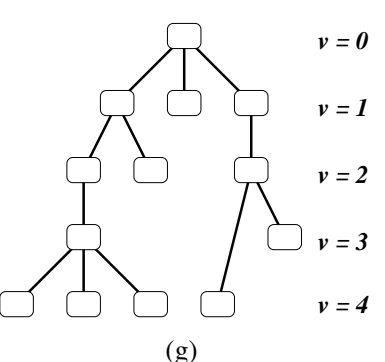

(g)
Fig. 1. (a) A grey-level image I : $[0,9]^{2} \rightarrow[0,4]$; (b-f) threshold images for $v$ varying from 0 (b) to 4 (f). (g) The component-tree of $I$.

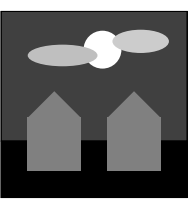

(a)

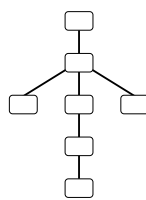

(b)

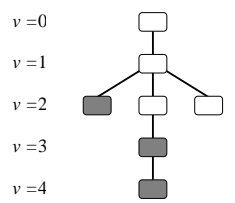

(c)

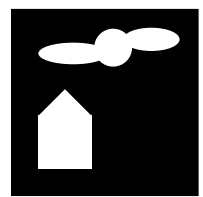

(d)
Fig. 2. (a) A grey-level image. (b) The component-tree $(\mathcal{K}, L, R)$ of (a) (c) A set of nodes $\widehat{\mathcal{K}} \subseteq \mathcal{K}$ (in grey). (d) Segmentation of (a) based on the set of nodes (c)

thresholding function $X_{v}: V^{E} \rightarrow 2^{E}$ by $X_{v}(I)=\{x \in E$ $v \leq I(x)\}$. For any $v \in V$ and any $X \subseteq E$, we define the cylinder function $C_{X, v}: E \rightarrow V$ by $C_{X, v}(x)=v$ if $x \in X$, and 0 otherwise. An image $I \in V^{E}$ can then be expressed as

$$
I=\bigvee_{v \in V} \bigvee_{X \in \mathcal{C}\left[X_{v}(I)\right]} C_{X, v}
$$

where $\bigvee$ is the pointwise supremum for the set of functions.

Let $\mathcal{K}=\bigcup_{v \in V} \mathcal{C}\left[X_{v}(I)\right]$ be the set of all the connected components obtained from the different thresholdings of $I$. The relation $\subseteq$ is a partial order on $\mathcal{K}$, and its Hasse diagram $(\mathcal{K}, L)$ is a tree, the root of which, called $R$ is $\sup (\mathcal{K}, \subseteq)=$ $E$. The rooted tree $T=(\mathcal{K}, L, R)$ is called component-tree of $I ; \mathcal{K}, R$ and $L$ are the set of the nodes, the root and the set of the edges of the tree, respectively. We set $\operatorname{ch}(N)=\left\{N^{\prime} \in\right.$ $\left.\mathcal{K} \mid\left(N, N^{\prime}\right) \in L\right\}, \operatorname{ch}(N)$ is the set of the children of the node $N$ in $T$. An example of component-tree is illustrated in Fig. 1.

\subsection{Segmentation based on component-trees}

Performing segmentation with a component-tree consists of correctly determining a subset $\widehat{\mathcal{K}} \subseteq \mathcal{K}$ of nodes. The binary resulting image $\widehat{I} \subseteq E$ is then defined as the union of the nodes of $\widehat{\mathcal{K}}$, i.e., $\widehat{I}=\bigcup_{N \in \widehat{\mathcal{K}}} N$. See Fig. 2 for an example.

The choice of $\widehat{\mathcal{K}}$ is generally based on properties ("attributes") stored at each node. The nodes are then chosen on local criteria. By opposition, the proposed approach consists of defining $\widehat{\mathcal{K}}$ in a global fashion, thanks to an example.

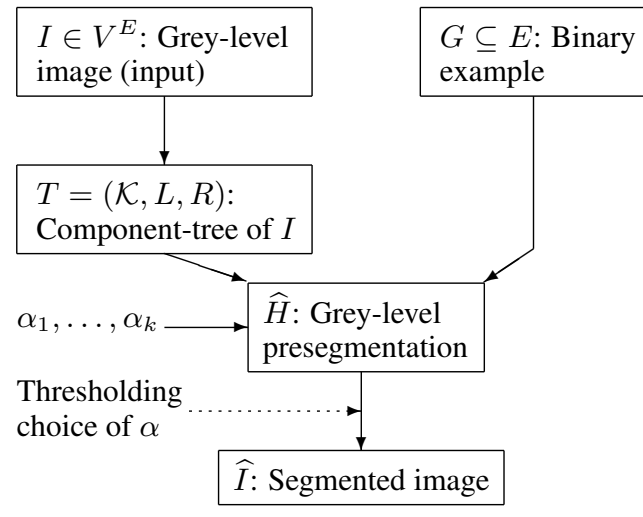

Fig. 3. Workflow of the example-based segmentation algorithm (see text).

\subsection{Example-based segmentation}

Given a segmentation example $G \subseteq E$, supposed to be a close approximation of the desired one, the purpose is to determine a set of nodes $\widehat{\mathcal{K}}$ (and then its associated segmentation $\widehat{I}$ ) which fits at best $G$, w.r.t. a distance modeling the ratio of false positives/negatives. Such a distance can be defined by

$$
d^{\alpha}(\widehat{I}, G)=\alpha|\widehat{I} \backslash G|+(1-\alpha)|G \backslash \widehat{I}|
$$

where $\alpha \in[0,1]$ controls the trade-off between false positives and false negatives tolerance. For a chosen $\alpha$, the purpose is then to solve the following minimization equation

$$
\widehat{\mathcal{K}}^{\alpha}=\arg \min _{\widehat{\mathcal{K}} \subseteq \mathcal{K}}\left\{d^{\alpha}(\widehat{I}, G)\right\}
$$

In [8], some of the authors have proved that $\widehat{\mathcal{K}}^{\alpha}=\mathcal{F}^{\alpha}(E)$, where $\mathcal{F}^{\alpha}$ is recursively defined, for all $N \in \mathcal{K}$, by

$$
\left\{\begin{array}{l}
\mathcal{F}^{\alpha}(N)=\{N\} \\
c^{\alpha}(N)=\alpha \cdot n(N, G)
\end{array}\right.
$$

if $\alpha \cdot n(N, G)<(1-\alpha) \cdot p^{*}(N, G)+\sum_{N^{\prime} \in c h(N)} c^{\alpha}\left(N^{\prime}\right)$ and

$$
\left\{\begin{array}{l}
\mathcal{F}^{\alpha}(N)=\bigcup_{N^{\prime} \in \operatorname{ch}(N)} \mathcal{F}^{\alpha}\left(N^{\prime}\right) \\
c^{\alpha}(N)=(1-\alpha) \cdot p^{*}(N, G)+\sum_{N^{\prime} \in \operatorname{ch}(N)} c^{\alpha}\left(N^{\prime}\right)
\end{array}\right.
$$

otherwise, where $p^{*}(N, G)$ is the number of points of $N \cap G$ which do not belong to any children of $N$, and $n(N, G)$ is the number of points of $N \backslash G$.

It can be proved that for $\alpha_{1}<\alpha_{2}$, we have $\widehat{I}^{\alpha_{2}} \subseteq \widehat{I}^{\alpha_{1}}$, where $\widehat{I}^{\alpha}=\bigcup_{N \in \widehat{\mathcal{K}}^{\alpha}} N$. Given a set $\left\{\alpha_{i}\right\}_{i=1}^{k}$ of increasing values, we can then generate a grey-level image $\widehat{H}$ such that for all $x \in E$

$$
\widehat{H}(x)=\bigvee_{i=1}^{k} C_{\widehat{I}^{\alpha_{i}, i}}(x)=\max \left\{i \in[1, k] \mid x \in I^{\alpha_{i}}\right\}
$$

The choice of a segmentation result $\widehat{I}$ can then be performed by the user by simply thresholding $\widehat{H}$ at a convenient value $\alpha_{i}$. The segmentation workflow is summarized in Fig. 3. 


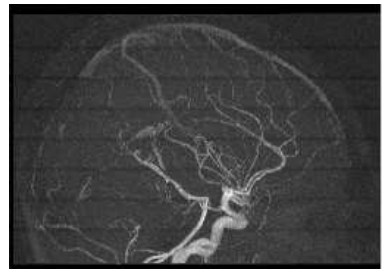

(a)

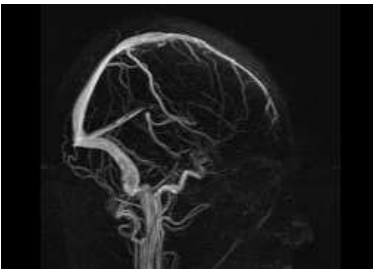

(b)
Fig. 4. MRA of the brain (same patient, sagittal MIP): (a) high resolution $(0.5 \mathrm{~mm})$ TOF MRA, and (b) low resolution (1mm) PC MRA.

\section{RESULTS}

In this section, experiments are performed in order to assess the relevance of the method. They deal with artery segmentation from (time of flight: TOF, and phase contrast: PC) MRA images visualizing both veins and arteries. Subsec. 3.1 focuses on the "component-tree" part of the method only, by assuming that the example is similar to the expected result and correctly positioned into the image (intra-patient cases). Subsec. 3.2 deals with the influence of the example quality, by assuming that it is not fully similar to the expected result and not perfectly positioned into the image (inter-patient cases).

\subsection{Intra-patient validations (TOF/PC MRA)}

We consider two images of a same patient: a high resolution $(0.5 \mathrm{~mm})$ TOF MRA and a low resolution $(1 \mathrm{~mm})$ PC MRA (Fig. 4). Two arterial ground-truths have been manually defined for both images (Fig. 5(a,e)).

In order to check the soundness of the method in the case where a "very good" example is provided, the segmentation of the TOF MRA has been carried out by using its own groundtruth as example. Some results (for various $\alpha$ values) are depicted in Fig. 5 (b-d). More quantitatively, Fig. 6 depicts the ROC (Receiver Operating Characteristic) curve (in red) induced by the computation of the results for $1000 \alpha$ values among $[0,1]$.

The case of a less satisfactory (but well positioned) example is considered by segmenting the same TOF MRA, but using the (less accurate) ground-truth of the PC MRA. Some results are depicted in Fig. $5(\mathrm{f}-\mathrm{h})$. Fig. 6 depicts the ROC curve (in blue) induced by the computation of these results.

This study tends to show the correct behavior of the method in case of correct and well positioned example. Note that the $\alpha$ value generating the best result depends on the example (and its trend to be a subset and/or a superset of the searched structure).

\subsection{Inter-patient validation (PC MRA)}

We now consider several low resolution $(1 \mathrm{~mm})$ PC MRAs (see Fig. 4(b)). We focus on the possible effects of interpatient variability. Indeed, the image to segment and the used example(s) are obtained from distinct patients.

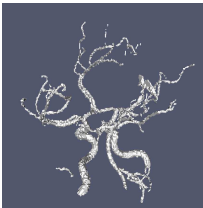

(a)

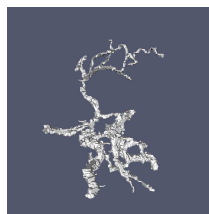

(e)

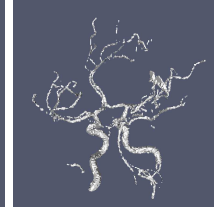

(b) $\alpha=0.00$

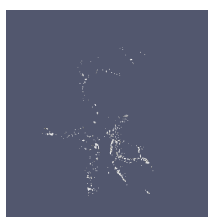

(f) $\alpha=0.00$

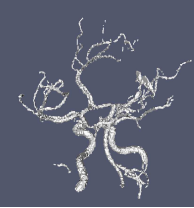

(c) $\alpha=0.50$

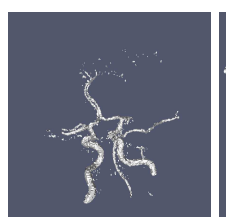

(g) $\alpha=0.50$

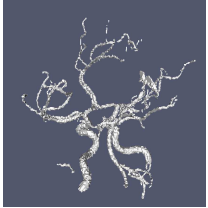

(d) $\alpha=0.95$

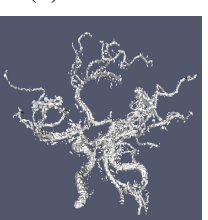

(h) $\alpha=0.95$
Fig. 5. (a) Ground-truth for the TOF MRA. (b-d) TOF MRA segmentations by using (a) as example. (e) Ground-truth for the PC MRA. (f-h) TOF MRA segmentations by using (e) as example.

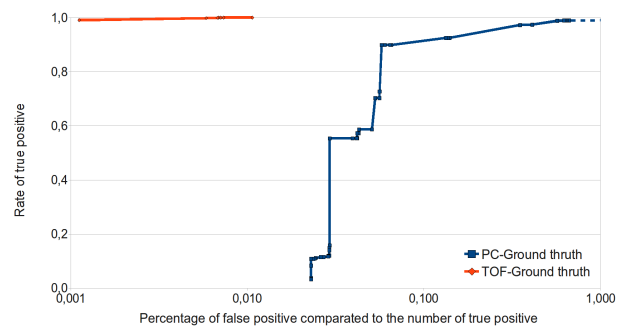

Fig. 6. ROC curves, for the two experiments of Subsec. 3.1 (logarithmic scale in horizontal axis).

The first effect is the inadequacy of the considered example with the image. Indeed, the example may be (more or less) morphologically, geometrically and topologically different from the vascular structures to segment.

A second effect is the (in)accuracy of the positioning of the example. Indeed, a registration step has to be carried out in order to put the example and the target image in a same referential. Despite recent works devoted to angiographic image registration $[9,10]$, the existing methods are still not reliable enough in the complex case of cerebral vessels. An alternative then remains, the "classic" registration on morphological images associated to the angiographic ones, as proposed here.

In order to qualitatively assess the behavior of the method w.r.t. these effects, two experiments are carried out by using as example a single segmented image or a "binary atlas" obtained from several segmented images. In each case, both affine and nonrigid registration [11] are considered.

First, the considered example is a single segmentation ground-truth. The results obtained for five different patients, are depicted Fig. 7. As expected, nonrigid registration provides better results than affine one (with a higher computational cost). With such registration, and despite the low quality of these images, we can globally obtain the Willis circle and its neighboring arteries.

Second, the considered example is a binary arterial atlas generated from 60 PC-MRAs. The results obtained for the same five patients as above, are depicted Fig. 8. Once again, 

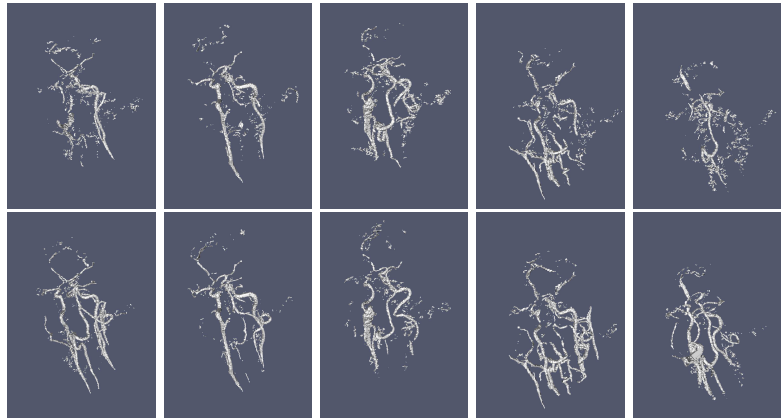

Fig. 7. Segmentation results $(\alpha=0.95)$ for 5 patients, with a single ground-truth as example (1st row: affine registration; 2nd row: nonrigid registration).
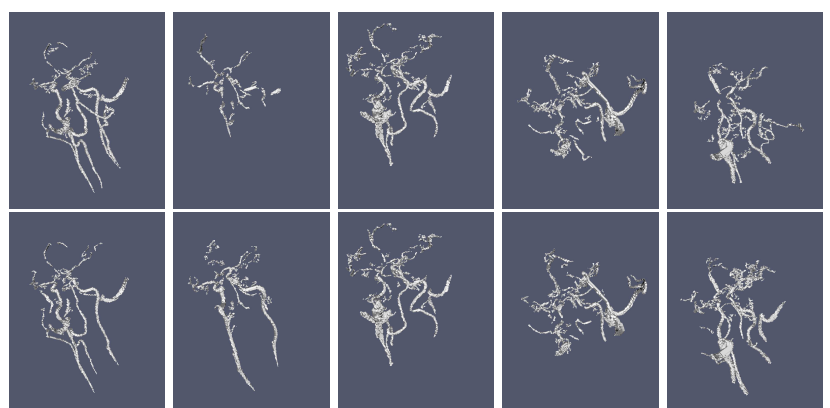

Fig. 8. Segmentation results $(\alpha=0.80)$ for 5 patients, with an atlas as example (1st row: affine registration; 2 nd row: nonrigid registration).

nonrigid registration results outperform affine ones. With such registration, the results are in particular much more accurate than those obtained with a single ground-truth, which probably relies on the fact that the atlas models more completely the anatomical variants which may appear among a population. (Note that with such an atlas, the tolerance to false positives must be higher than with a single ground-truth, implying a different value for $\alpha$.)

\section{CONCLUSION}

A method relying on example-based segmentation and component-trees has been proposed for assisted segmentation of vessels from 3D data. First experiments tend to show the relevance of the approach. They also emphasize remaining weaknesses, and the need for further improvements, especially related to registration and example quality. Further works will consist of $(i)$ developing hybrid morphology/angiography registration methods, better suited for complex vessels, and $(i i)$ proposing more efficient examples (for instance statistical, instead of binary ones). More straightforward extensions of this work will be the development a fully interactive version of the segmentation method (relying on presegmentations on MIP images) and the use of a preprocessing [12] to enhance the vesselness of the images, which is a strong requirement for connected filtering approaches.

\section{REFERENCES}

[1] D. Lesage, E. D. Angelini, I. Bloch, and G. Funka-Lea, "A review of 3D vessel lumen segmentation techniques: Models, features and extraction schemes," Medical Image Analysis, vol. 13, no. 6, pp. 819-845, 2009.

[2] Y. Schnitman, Y. Caspi, D. Cohen, and D. Lischinski, "Inducing semantic segmentation from an example," in ACCV, 2006, vol. 3852 of LNCS, pp. 373-384.

[3] C. Gallagher and A. Kokaram, "Bayesian example based segmentation using a hybrid energy model," in ICIP, 2007, pp. 41-44.

[4] S. Faisan, N. Passat, V. Noblet, R. Chabrier, J.-P. Armspach, and C. Meyer, "Segmentation of head bones in 3-D CT images from an example," in ISBI, 2008, pp. $81-84$.

[5] B. Bouraoui, C. Ronse, J. Baruthio, N. Passat, and P. Germain, "3D segmentation of coronary arteries based on advanced Mathematical Morphology techniques," Computerized Medical Imaging and Graphics, vol. 34, no. 5, pp. 377-387, 2010.

[6] P. Salembier, A. Oliveras, and L. Garrido, "Antiextensive connected operators for image and sequence processing," IEEE Transactions on Image Processing, vol. 7, no. 4, pp. 555-570, 1998.

[7] B. Caldairou, N. Passat, and B. Naegel, "Attributefiltering and knowledge extraction for vessel segmentation," in ISVC, 2010, vol. 6453 of LNCS, pp. 13-22.

[8] N. Passat and B. Naegel, "Selection of relevant nodes from component-trees in linear time," in DGCI, Submitted (2011).

[9] W. H. Nam, D.-G. Kang, D. Lee, and J. B. Ra, "Robust registration of $3 \mathrm{D}$ ulstrasound and CT images of the liver for image-guided intervention," in ISBI, 2010, pp. 1201-1204.

[10] J. W. Suh, D. Scheinost, X. Qian, A. J. Sinusas, C. K. Breuer, and X. Papademetris, "Serial non rigid vascular registration using weighted normalized mutual information," in ISBI, 2010, pp. 25-28.

[11] V. Noblet, C. Heinrich, F. Heitz, and J.-P. Armspach, “3$\mathrm{D}$ deformable image registration: a topology preservation scheme based on hierarchical deformation models and interval analysis optimization," IEEE Transactions on Image Processing, vol. 14, no. 5, pp. 553-566, 2005.

[12] O. Tankyevych, H. Talbot, P. Dokládal, and N. Passat, "Direction-adaptive grey-level morphology. Application to 3D vascular brain imaging," in ICIP, 2009, pp. 22612264. 